\title{
Critical role for GLP-1 in symptomatic post-bariatric hypoglycaemia
}

\author{
Colleen M. Craig ${ }^{1} \cdot$ Li-Fen Liu ${ }^{1}$ - Carolyn F. Deacon ${ }^{2} \cdot$ Jens J. Holst $^{2}$ • \\ Tracey L. McLaughlin ${ }^{1}$
}

Received: 9 September 2016 / Accepted: 23 November 2016 / Published online: 14 December 2016

(C) Springer-Verlag Berlin Heidelberg 2016

\begin{abstract}
Aims/hypothesis Post-bariatric hypoglycaemia $(\mathrm{PBH})$ is a rare, but severe, metabolic disorder arising months to years after bariatric surgery. It is characterised by symptomatic postprandial hypoglycaemia, with inappropriately elevated insulin concentrations. The relative contribution of exaggerated incretin hormone signalling to dysregulated insulin secretion and symptomatic hypoglycaemia is a subject of ongoing inquiry. This study was designed to test the hypothesis that $\mathrm{PBH}$ and associated symptoms are primarily mediated by glucagon-like peptide-1 (GLP-1).

Methods We conducted a double-blinded crossover study wherein eight participants with confirmed PBH were assigned in random order to intravenous infusion of the GLP-1 receptor (GLP-1r) antagonist. Exendin (9-39) (Ex-9), or placebo during an OGTT on two separate days at the Stanford University Clinical and Translational Research Unit. Metabolic, symptomatic and pharmacokinetic variables were evaluated. Results were compared with a cohort of BMI- and glucosematched non-surgical controls (NSCs).

Results Infusion of Ex-9 decreased the time to peak glucose and rate of glucose decline during OGTT, and raised the
\end{abstract}

Electronic supplementary material The online version of this article (doi:10.1007/s00125-016-4179-x) contains peer-reviewed but unedited supplementary material, which is available to authorised users.

Colleen M. Craig

cmcraig@stanford.edu

1 Department of Medicine, Division of Endocrinology, Stanford University School of Medicine, 300 Pasteur Drive, Room S025, Stanford, CA 94305, USA

2 Department of Biomedical Sciences, Panum Institute, University of Copenhagen, Copenhagen, Denmark postprandial nadir by over $70 \%$, normalising it relative to NSCs and preventing hypoglycaemia in all PBH participants. Insulin AUC and secretion rate decreased by $57 \%$ and $71 \%$ respectively, and peak postprandial insulin was normalised relative to NSCs. Autonomic and neuroglycopenic symptoms were significantly reduced during Ex-9 infusion.

Conclusions/interpretation GLP-1r blockade prevented hypoglycaemia in $100 \%$ of individuals, normalised beta cell function and reversed neuroglycopenic symptoms, supporting the conclusion that GLP-1 plays a primary role in mediating hyperinsulinaemic hypoglycaemia in PBH. Competitive antagonism at the GLP-1r merits consideration as a therapeutic strategy. Trial registration: ClinicalTrials.gov NCT02550145

Keywords Bariatric complication - GLP-1 . Hyperinsulinaemic hypoglycaemia $\cdot$ Hypoglycaemia $\cdot$ Late dumping syndrome $\cdot$ Nesidioblastosis $\cdot$ Neuroglycopenia . NIPHS · Noninsulinoma pancreatogenous hypoglycaemia syndrome $\cdot$ Post-bariatric hypoglycaemia $\cdot$ Roux-en-Y gastric bypass $\cdot$ RYGB

$\begin{array}{ll}\text { Abbreviations } \\ \text { EHSS } & \text { Edinburgh Hypoglycaemia Symptom Scale } \\ \text { Ex-9 } & \text { Exendin (9-39) } \\ \text { GIP } & \text { Gastric inhibitory polypeptide } \\ \text { GLP-1 } & \text { Glucagon-like peptide 1 } \\ \text { GLP-1r } & \text { GLP-1 receptor } \\ \text { IGI } & \text { Insulinogenic index } \\ \text { ISR } & \text { Insulin secretion rate } \\ \text { NSC } & \text { Non-surgical control } \\ \text { PBH } & \text { Post-bariatric hypoglycaemia } \\ \text { RYBG } & \text { Roux-en-Y gastric bypass }\end{array}$




\section{Introduction}

More than one-third, or 78.6 million, US adults are obese [1], with annual costs of $\$ 147$ billion in 2008 [2]. Lifestyle and medical therapies yield modest and often temporary weight loss. The need for more extensive and permanent weight loss strategies has fuelled the popularity of surgical weight loss interventions. Bariatric surgery is currently performed on approximately 200,000 Americans annually, producing longterm weight loss of 50-70\% excess body weight, major reductions in clinical morbidities and decreased death from multiple causes [3, 4]. Roux-en-Y gastric bypass (RYGB), comprising over one-third of all procedures, cures diabetes in $83 \%$ of individuals [5, 6]. With the recent addition of bariatric surgery to the treatment algorithm for type 2 diabetes [7], the use of RYGB may rise.

The physiological mechanisms mediating the resolution of diabetes after RYGB are controversial, as glycaemic improvement occurs independent of weight loss, and is significantly greater than that observed with medical therapy [8] or gastric banding [9]. Indeed, both duodenal exclusion, leading to decreased stimulation of a pro-diabetic 'foregut factor,' vs rapid delivery of nutrients to the ileum, leading to increased stimulation of an anti-diabetic 'hindgut factor', have been proposed as causes of early diabetes resolution after RYGB. The latter relates to postoperative increases in secretion of hindgut-derived glucagon-like peptide-1 (GLP-1) as key mediator of glycaemic reductions after bariatric surgery [10]. However, the role of GLP-1 continues to be challenged [11].

In $0.2-6.6 \%$ of all RYGB procedures [12-14], severe symptomatic hypoglycaemia results. This disorder leads to frequent episodes of postprandial hypoglycaemia, with glucose concentrations low enough to cause seizures, loss of consciousness, cognitive dysfunction, disability and death. The threat of neuroglycopenia is worsened by a high prevalence of hypoglycaemia unawareness, with one study demonstrating that continuous glucose monitoring over 5 days detected hypoglycaemic episodes of $<3.05 \mathrm{mmol} / \mathrm{l}$ in $75 \%$ of post-RYGB patients [15]. There are no approved pharmacotherapies and treatment typically rests on dietary changes, including frequent small meals with dietary carbohydrate restriction. A stepped pharmacotherapy approach follows (acarbose, octreotide, diazoxide), for which some evidence exists [16-18]. However, these medications are limited by poor efficacy and tolerability. Refractory patients are left with surgical options, such as insertion of a gastrostomy tube to the remnant stomach [19, 20], placement of a gastric band or gastrojejunal anastomotic reduction to slow transit [21] or RYGB reversal, which has shown unreliable results [22]. Refractory patients have undergone total pancreatectomy, resulting in insulindependent diabetes and carrying over a $6 \%$ operative mortality risk [23].
The cause for this disturbed metabolism is not known, but it may represent an exaggeration of the same physiological changes mediating the early resolution of type 2 diabetes after bariatric surgery. Post-bariatric hypoglycaemia ( $\mathrm{PBH})$ is characterised by inappropriately high insulin concentrations when glucose is low [24], occurring 1-3 h after oral nutrient ingestion, and while insulin concentrations are typically normal after overnight fasting or in response to intravenous glucose, high postprandial concentrations are out of proportion to the degree of both glucose elevation and insulin resistance [25]. Early reports attributed glucose lowering to beta cell hypertrophy, hyperplasia and hypersecretion [24, 26]. Accumulating evidence points to altered nutrient transit and exaggerated secretion of (and/or sensitivity to) GLP-1. Studies have demonstrated accelerated gastric emptying caused by altered nutrient transit following RYGB [27], as well as a strong correlation between postprandial GLP-1 and rates of gastric emptying [28]. Indeed, postprandial concentrations of intact GLP-1 are up to tenfold higher after RGYB in asymptomatic patients, and are even more pronounced in symptomatic patients with $\mathrm{PBH}[9,29]$.

There have been few studies involving patients with PBH. We have previously shown that delivery of an isoglycaemic standardised liquid meal to the remnant stomach via gastrostomy tube (in which nutrients transit via the foregut) vs oral delivery (in which nutrients bypass the foregut) reduced glycaemic excursions and GLP-1 concentrations [19, 20], pointing to a central role for enhanced incretin effect as a result of altered nutrient transit. GLP-1, secreted by L-cells in the ileum and hindgut, is favoured as the primary incretin contributor because it is more consistently elevated following RYGB than gastric inhibitory polypeptide (GIP) [30], which is secreted primarily by the foregut. In concert with having higher postprandial GLP-1, symptomatic patients also exhibit higher postprandial insulin as measured by insulin/glucose ratio [29], insulinogenic index (IGI) [31] or beta cell glucose sensitivity [32]. These suggest that an exaggerated GLP-1 response and/or robust insulin response to GLP-1 may contribute. Indeed, it has been shown that at very high GLP-1 concentrations insulin secretion is augmented by a dosedependent potentiation of the dose-response relationship between plasma glucose and insulin secretion [33].

Investigations evaluating the glucose-lowering influences of GLP-1 post-RYGB surgery, however, are few, and the subject remains controversial $[10,11]$. We designed a study to further evaluate the role of GLP-1 in mediating: (1) hyperinsulinaemic hypoglycaemia; and (2) the presence and severity of autonomic and neuroglycopenic symptoms, by infusing placebo (normal $\mathrm{NaCl}$ solution) or the GLP-1r antagonist, exendin (9-39) (Ex-9) during an OGTT in patients with symptomatic PBH compared with BMI and glucose-matched non-surgical controls (NSCs). This study contributes to the small body of literature conducted to date by only a single 
investigator evaluating the role of GLP-1 in PBH, and is the first investigation to directly address the ability of GLP-1 receptor (GLP-1r) blockade to improve symptoms of hypoglycaemia - a concern of high clinical relevance in this patient population.

\section{Methods}

\section{Study design}

This was a double-blinded crossover study conducted at the Stanford University School of Medicine Clinical and Translational Research Unit. All PBH participants were assigned to receive Ex-9 or placebo infusion during an OGTT on 2 days separated by 1-7 days, in random order. The protocol was approved by the Stanford University Institutional Review Board, registered with clinicaltrials.gov (ClinicalTrial.gov registration no. NCT02550145), and conducted after Food and Drug Administration (FDA) review as an investigational new drug (IND 105018). All participants provided written informed consent prior to participation in the study, which was carried out in accordance with the principles of the Declaration of Helsinki as revised in 2008.

\section{Participant characteristics}

PBH participants Ten post-RYGB individuals had: (1) Whipple's triad by history (symptomatic hypoglycaemia, capillary glucose $\leq 3.1 \mathrm{mmol} / 1$, with symptom resolution by carbohydrate administration); and (2) occurrence of capillary glucose $\leq 2.78 \mathrm{mmol} / 1$ at least once per month by patient report. Of these, eight participants developed a plasma glucose $\leq 3.1 \mathrm{mmol} / 1$ during OGTT with placebo infusion, in association with inappropriately elevated plasma insulin $\geq 21 \mathrm{pmol} / 1$ or C-peptide $>0.1 \mathrm{nmol} / \mathrm{l}$.

NSC participants From our database of 530 non-surgical nondiabetic individuals, an NSC was selected for each $\mathrm{PBH}$ participant on the basis of matching for BMI, age, sex and peak postprandial glucose, because of its stimulatory effect on insulin secretion. As postprandial glucose is often in the diabetic range despite normal fasting glucose in post-RYGB individuals, it is challenging to match non-diabetic controls for peak postprandial glucose. Thus, while peak glucose was slightly lower, the controls chosen were the closest matches identified.

\section{Experimental protocols}

OGTT procedure On each study day, participants arrived after a $12 \mathrm{~h}$ fast. Weight and vital signs were obtained, a catheter was inserted into the antecubital vein of each arm and a fasting blood sample was drawn at T-31 min. At T-30, an intravenous priming bolus of Ex-9 (7500 pmol/kg) or placebo was administered over $1 \mathrm{~min}$, followed by continuous infusion at a rate of $500 \mathrm{pmol} \mathrm{kg}^{-1} \mathrm{~min}^{-1}$ for $210 \mathrm{~min}$. At $\mathrm{T}=0$, patients consumed a $75 \mathrm{~g}$ glucola drink, divided into quarters, with each quarter given over $5 \mathrm{~min}$, for a total of $20 \mathrm{~min}$. Over $180 \mathrm{~min}$, plasma samples were drawn every $30 \mathrm{~min}$. The rate of sampling doubled if the plasma glucose fell below $5.55 \mathrm{mmol} / \mathrm{l}$, with intermediary draws as glucose approached $2.78 \mathrm{mmol} / 1$. At $2.78 \mathrm{mmol} / \mathrm{l}$ or less, the test was stopped and investigators intervened as needed to normalise glucose. Participants were blinded to all glucose results.

Hypoglycaemia symptom questionnaire To assess the temporal presence and severity of autonomic, neuroglycopenic and non-specific symptoms, we imposed a five-point Likert severity gradation ( $0=$ none; $5=$ severe) on the Edinburgh Hypoglycaemia Symptom Scale (EHSS) - a survey validated for use in insulin-treated diabetic patients for identification of symptoms of acute hypoglycaemia [34, 35]. The severitygraded EHSS was completed by participants every $30 \mathrm{~min}$ prior to each blood draw during each OGTT. Symptoms assessed included autonomic (sweating, shaking, palpitations, hunger), neuroglycopenic (blurred vision, confusion, drowsiness, odd behaviour, speech difficulty, incoordination, dizziness, inability to concentrate) and malaise (nausea, headache). For each participant, a composite score for 'all timepoints' was recorded. To isolate symptoms associated temporally with the glucose rise period (from $\mathrm{T}=0$ to the individual participants' glucose peak) and the glucose fall period (from glucose peak to nadir), two sub-scores were included, 'glucose rise' and 'glucose fall' scores. Analysis further grouped symptom scores according to symptom type: autonomic, neuroglycopenic or malaise.

Peptide and assays Ex-9 acetate was purchased as lyophilised sterile powder from Bachem (Clinalfa, Läufelfingen, Switzerland) and stored at $-20^{\circ} \mathrm{C}$ in the Stanford Investigational Drug Pharmacy. On the day of Ex-9 infusion, $10 \mathrm{mg}$ vials of peptide were diluted in the appropriate volume of $0.9 \% \mathrm{NaCl}(154 \mathrm{mmol} / \mathrm{l})$ and $25 \%$ human serum albumin $(250 \mathrm{~g} / \mathrm{l})$ was added to prevent peptide adhesion. Low-sorbing bags and tubing were used. Plasma samples were placed on ice, centrifuged, aliquotted and stored at $-20^{\circ} \mathrm{C}$ until assayed. Glucose concentrations were determined immediately by glucose oxidase method (Analyzer 2; Beckman, Brea, CA, USA). Insulin, C-peptide and glucagon were measured by radioimmunoassay according to the manufacturer's specifications (Millipore, St Charles, MO, USA). Total GLP-1 and GIP concentrations were determined by radioimmunoassay using C-terminally-directed antisera (numbers 89390 and 80867) [36, 37]. Ex-9 was measured using antibody 3145 raised in rabbits immunised with exendin-4, which shows $100 \%$ cross-reactivity with Ex-9 but $0.01 \%$ cross-reactivity with GLP-1, glucagon or GIP [38]. 
Calculations and analysis Insulin sensitivity was estimated by HOMA-IR [39]. IGI was calculated as the ratio of incremental insulin to incremental plasma glucose from 0 to $30 \mathrm{~min}$ during the OGTT. Disposition index was calculated as IGI/HOMA-IR [40]. Insulin secretion rate (ISR) was calculated through deconvolution of peripheral $\mathrm{C}$-peptide concentrations using a two-compartment model of C-peptide kinetics [41] and population-based C-peptide kinetics with adjustment for age, sex and BMI [42]. ISR adjusted for glucose stimulus $\left(\mathrm{ISR}_{2 \mathrm{~h}} /\right.$ $\mathrm{G}_{2 \mathrm{~h}}$ ) was derived by taking the ratio of the integral of insulin secretion to the integral of glucose concentration over the first $120 \mathrm{~min}$ [43]. AUC calculations were performed using the trapezoidal rule. Because of the potential cancelling effect of the early rise and late fall in plasma glucose and insulin when considered as AUC over $180 \mathrm{~min}$, AUCs were partitioned into 0-60, 60-180 and 0-180 min. Insulin clearance was calculated as $\mathrm{AUC}_{\mathrm{ISR}} / \mathrm{AUC}_{\text {insulin. }}$ The rate of glucose decline was calculated as $\left(\right.$ glucose $_{\text {peak }}-$ glucose $\left._{\text {peak }+30 \mathrm{~min}}\right) / 30 \mathrm{~min}$. When the test was stopped early because of hypoglycaemia, the last data point recorded was carried forward.

Statistical analysis Data are presented as mean \pm SEM. Twotailed paired Student's $t$ tests were used for intra-group comparisons for placebo vs Ex-9. Two-tailed unpaired Student's $t$ tests were used for between-group differences. A $p$ value $<0.05$ was considered statistically significant. Data were graphed using Prism software (GraphPad, La Jolla, CA, USA).

\section{Results}

\section{Baseline characteristics of study cohorts}

Study cohorts included eight participants with $\mathrm{PBH}$, and eight age-, sex-, BMI- and glucose-matched NSCs (Table 1). Age and BMI (postoperative for the surgical cohort) did not differ between groups, and all participants were female. On average, the surgical cohort was 5 years postoperative and experienced

Table 1 Characteristics of PBH participants and NSCs

\begin{tabular}{llll}
\hline Characteristic & PBH $(n=8)$ & NSC $(n=8)$ & $p$ value \\
\hline Age (years) & $46 \pm 4$ & $47 \pm 3$ & 0.802 \\
Sex (male/female) & $0 / 8$ & $0 / 8$ & NA \\
BMI $\left(\mathrm{kg} / \mathrm{m}^{2}\right)$ & $31 \pm 2$ & $31 \pm 0$ & 0.999 \\
Systolic BP $(\mathrm{mmHg})$ & $120 \pm 4$ & $129 \pm 8$ & 0.332 \\
Diastolic BP (mmHg) & $76 \pm 2$ & $76 \pm 7$ & 0.955 \\
History of T2D (yes/no) & $0 / 8$ & $0 / 8$ & NA \\
HOMA-IR & $0.9 \pm 0.2$ & $3.7 \pm 0.4$ & $<0.001$ \\
\hline
\end{tabular}

Data are presented as mean $\pm \mathrm{SEM}$

$p$ values as assessed using the unpaired Student's $t$ test

NA, not applicable; T2D, type 2 diabetes a first documented episode of symptomatic hypoglycaemia 2 years postoperatively. All $\mathrm{PBH}$ participants experienced hypoglycaemic episodes with capillary glucose $<2.78 \mathrm{mmol} /$ 1 at least monthly, with the majority reporting at least weekly $(88 \%)$ or daily $(63 \%)$ episodes. Half reported loss of consciousness at least monthly and $63 \%$ reported seizure at least monthly (electronic supplementary material [ESM] Table 1).

\section{Metabolic response to OGTT during placebo infusion in PBH vs NSC participants}

Glucose As shown in Table 2 and Fig. 1a, fasting glucose did not differ between cohorts, but peak glucose was significantly greater in the bariatric cohort $(p<0.001)$. All PBH participants became hypoglycaemic during placebo, requiring rescue at plasma glucose $\leq 2.78 \mathrm{mmol} / 1$, whereas NSC participants remained normoglycaemic. The drop from peak to nadir glucose was greater for $\mathrm{PBH}$ than NSC participants $(p<0.001)$, despite the fact that the natural nadir for $\mathrm{PBH}$ participants would have been lower had the investigators not intervened for safety. The rate of glucose decline from peak to 60 min post peak was greater in $\mathrm{PBH}$ vs controls $(p<0.01)$. $\mathrm{AUC}_{\mathrm{glucose}}$ was greater for $\mathrm{PBH}$ than NSC participants during the 0-60 min interval and significantly lower than NSC during the 60-180 min interval, within which time all $\mathrm{PBH}$ participants became hypoglycaemic.

Insulin As shown in Table 2 and Fig. 1b, despite BMI matching, fasting insulin in $\mathrm{PBH}$ participants was nearly $75 \%$ lower than that of NSCs $(p<0.001)$, who were markedly more insulin resistant than the $\mathrm{PBH}$ group $(p<0.001)$. In contrast, early (0-60 $\mathrm{min}$ ) insulin concentrations were $181 \%$ higher in the surgical vs the NSC cohort $(p=0.005)$, with peak insulin over twice that of NSCs. Insulin concentrations rose by 50 -fold in the PBH group vs by 5.7 -fold in NSCs. These dramatic swings in insulin levels in $\mathrm{PBH}$ participants were not reflected

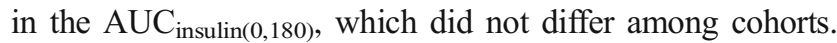
However, $\mathrm{AUC}_{\text {Insulin(0,60) }}$ was nearly twofold greater in $\mathrm{PBH}$ vs NSC participants. IGI, a measurement of insulin secretion relative to glucose concentration, did not differ significantly in PBH vs controls. Adjustment for insulin sensitivity, however, as reflected in the disposition index, demonstrated a sixfold higher beta cell response. For all $\mathrm{PBH}$ participants, insulin at the time of hypoglycaemia was inappropriately elevated, with an average plasma concentration of $121 \mathrm{pmol} / \mathrm{l}$.

\section{Metabolic response to OGTT during placebo vs Ex-9 infusion in $\mathrm{PBH}$ participants}

Glucose As shown in Table 2 and Fig. 1a, fasting plasma glucose concentrations were unchanged by $30 \mathrm{~min}$ Ex-9 infusion. Peak glucose values were unchanged, but time to peak glucose was accelerated by almost 20 min during GLP-1r blockade 
Table 2 Metabolic responses to OGTT during i.v. infusion of placebo vs Ex-9 in PBH participants and normalisation of metabolic variables compared with matched NSCs

\begin{tabular}{|c|c|c|c|c|c|c|}
\hline \multirow[t]{2}{*}{ Variable } & \multicolumn{2}{|l|}{$\mathrm{PBH}(n=8)$} & \multirow[t]{2}{*}{$\mathrm{NSC}(n=8)$} & \multicolumn{3}{|c|}{ Statistical effects } \\
\hline & Placebo & Ex-9 & & $p^{\mathrm{a}}$ & $p^{\mathrm{b}}$ & $p^{\mathrm{c}}$ \\
\hline \multicolumn{7}{|l|}{ Glucose (mmol/l) } \\
\hline Fasting & $5.1 \pm 0.1$ & $5.3 \pm 0.2$ & $5.6 \pm 0.2$ & 0.414 & 0.065 & 0.370 \\
\hline Peak & $13.1 \pm 0.6$ & $12.5 \pm 0.8$ & $8.4 \pm 0.3$ & 0.432 & $<0.001$ & 0.001 \\
\hline Time to peak (min) & $56 \pm 4$ & $38 \pm 5$ & $45 \pm 6$ & 0.011 & 0.223 & 0.226 \\
\hline Nadir & $2.6 \pm 0.1$ & $4.4 \pm 0.3$ & $4.2 \pm 0.2$ & $<0.001$ & $<0.001$ & 0.990 \\
\hline Time to hypoglycaemia (min) & $138 \pm 5$ & NA & NA & NA & NA & NA \\
\hline$\Delta$ peak-nadir & $10.5 \pm 0.6$ & $8.1 \pm 0.8$ & $4.3 \pm 0.4$ & 0.012 & $<0.001$ & 0.001 \\
\hline Rate of loss $\left(\mathrm{mmol} \mathrm{l}^{-1} \mathrm{~min}^{-1}\right)$ & $0.17 \pm 0.03$ & $0.06 \pm 0.02$ & $0.03 \pm 0.01$ & 0.001 & 0.001 & 0.189 \\
\hline \multicolumn{7}{|l|}{$\operatorname{AUC}(\mathrm{mmol} / \mathrm{l} \mathrm{min})$} \\
\hline 0-60 min & $577.3 \pm 17.2$ & $605.3 \pm 34.6$ & $450.7 \pm 15.9$ & 0.278 & $<0.001$ & 0.001 \\
\hline $60-180 \mathrm{~min}$ & $628.2 \pm 31.8$ & $854.6 \pm 65.5$ & $740.7 \pm 28.0$ & $<0.001$ & 0.019 & 0.133 \\
\hline $0-180 \mathrm{~min}$ & $1205.5 \pm 38.9$ & $1459.8 \pm 99.1$ & $1191.4 \pm 35.6$ & 0.020 & 0.793 & 0.023 \\
\hline \multicolumn{7}{|l|}{ Insulin (pmol/1) } \\
\hline Fasting & $27.6 \pm 133.3$ & $22.5 \pm 5.7$ & $104.2 \pm 8.2$ & 0.260 & $<0.001$ & $<0.001$ \\
\hline Peak & $1390.8 \pm 197.8$ & $612.9 \pm 159.6$ & $597.3 \pm 57.8$ & $<0.001$ & $<0.001$ & 0.870 \\
\hline Time to peak (min) & $60.0 \pm 6$ & $48.8 \pm 5$ & $67.5 \pm 12$ & 0.285 & 0.311 & 0.102 \\
\hline \multicolumn{7}{|l|}{$\mathrm{AUC}(\mathrm{pmol} / 1 \mathrm{~min})$} \\
\hline $0-60 \mathrm{~min}$ & $43,200 \pm 5322$ & $23,397 \pm 5781$ & $23,751 \pm 2610$ & 0.001 & 0.005 & 0.956 \\
\hline $60-180 \mathrm{~min}$ & $31,905 \pm 6086$ & $17,100 \pm 3645$ & $45,368 \pm 4222$ & 0.050 & 0.091 & $<0.001$ \\
\hline $0-180 \mathrm{~min}$ & $94,490 \pm 12,633$ & $40,497 \pm 8897$ & $69,120 \pm 6041$ & 0.001 & 0.092 & 0.019 \\
\hline \multicolumn{7}{|l|}{ Beta cell function } \\
\hline Insulin at glucose $\leq 3.1 \mathrm{mmol} / 1$ & $121.4 \pm 32.8$ & NA & NA & NA & NA & NA \\
\hline $\mathrm{IGI}_{30}$ & $0.5 \pm 0.1$ & $0.3 \pm 0.1$ & $0.5 \pm 0.1$ & 0.001 & 0.927 & 0.286 \\
\hline Disposition index $_{0-30}$ & $0.2 \pm 0.5$ & $0.2 \pm 0.5$ & $1.5 \pm 0.1$ & 0.002 & 0.006 & 0.061 \\
\hline $\mathrm{ISR}_{2 \mathrm{~h}} / \mathrm{G}_{2 \mathrm{~h}}$ & $9.4 \pm 1.2$ & $3.9 \pm 0.4$ & NA & 0.001 & NA & NA \\
\hline \multicolumn{7}{|l|}{ C-peptide (nmol/l) } \\
\hline Fasting & $2.2 \pm 0.2$ & $2.4 \pm 0.2$ & NA & 0.239 & NA & NA \\
\hline $\mathrm{AUC}_{0-180}(\mathrm{nmol} / \mathrm{l} \mathrm{min})$ & $3610.2 \pm 391$ & $1755.8 \pm 215$ & NA & $<0.001$ & NA & NA \\
\hline \multicolumn{7}{|l|}{ ISR $\left(\mathrm{pmol} \mathrm{kg}^{-1} \min ^{-1}\right)$} \\
\hline Fasting & $6.5 \pm 0.5$ & $7.0 \pm 0.7$ & NA & 0.203 & NA & NA \\
\hline Peak & $170.0 \pm 57.7$ & $50.2 \pm 7.6$ & NA & 0.001 & NA & NA \\
\hline \multicolumn{7}{|l|}{ Insulin clearance } \\
\hline Fasting & $2.5 \pm 0.6$ & $4.1 \pm 1.3$ & NA & 0.082 & NA & NA \\
\hline Postprandial $_{0-180}$ & $0.9 \pm 0.1$ & $0.73 \pm 0.2$ & NA & 0.080 & NA & NA \\
\hline
\end{tabular}

Data are presented as mean \pm SEM

${ }^{a} \mathrm{PBH}$ placebo vs Ex-9, paired two-tailed Student's $t$ test

${ }^{\mathrm{b}} \mathrm{PBH}$ placebo vs NSC, paired two-tailed Student's $t$ test

${ }^{\mathrm{c}} \mathrm{PBH}$ Ex-9 vs NSC, unpaired two-tailed Student's $t$ test

NA, not appropriate

$(p<0.05)$. Postprandial glucose nadir was raised by over $70 \%$, and normalised relative to NSCs. Hypoglycaemia was prevented in all eight PBH individuals. In addition, both the magnitude of the glucose drop and the rate of glucose decline were significantly reduced by Ex-9 $(p<0.001)$, with $\mathrm{AUC}_{\text {glucose }(60,180)}$ normalised relative to NSC.
Insulin The insulin spike occurring approximately $1 \mathrm{~h}$ postprandially in $\mathrm{PBH}$ participants during placebo was prevented by Ex-9 in 100\% of individuals (insulin 1390 to $612 \mathrm{pmol} / \mathrm{l}$ ), yielding peak insulin concentrations comparable with those of controls. Total $\mathrm{AUC}_{\text {insulin(0,180) }}$ decreased by $57 \%$, and was significantly lower than controls 

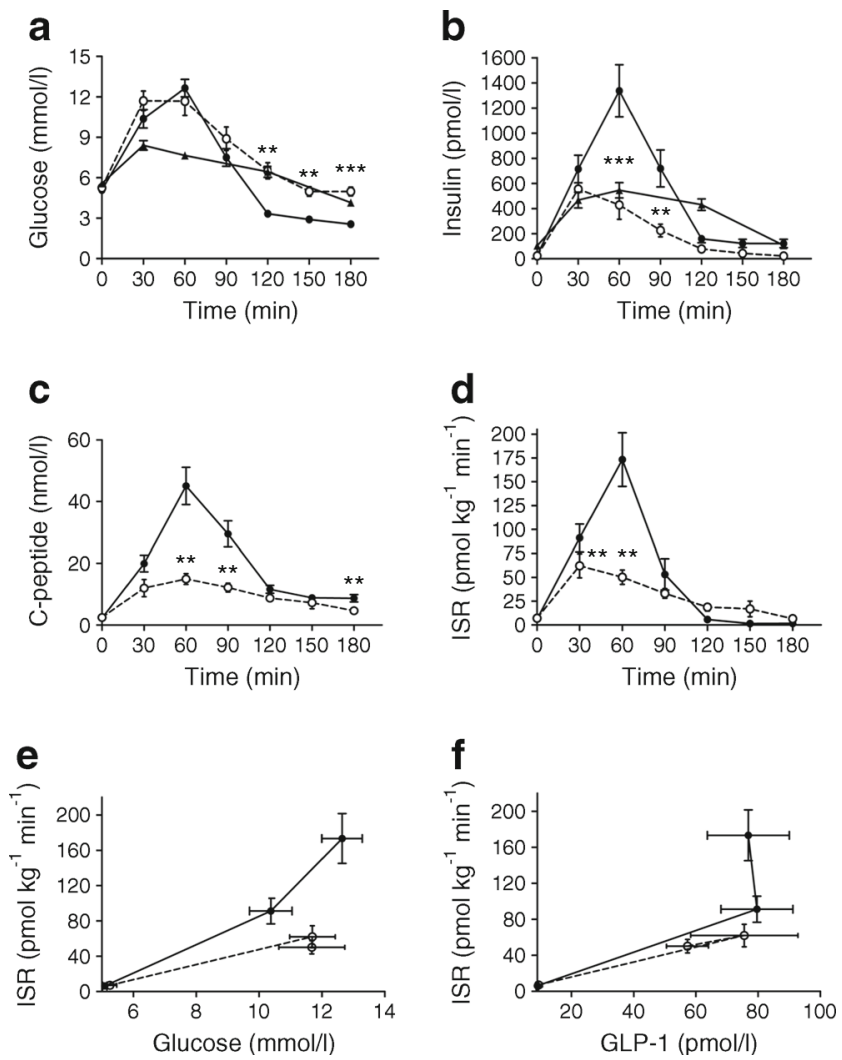

Fig. 1 Plasma glucose (a), insulin (b), C-peptide (c) and ISR (d) in response to OGTT in participants with $\mathrm{PBH}$ during placebo (solid line, black circles) vs Ex-9 (dashed line, white circles) infusion, compared with NSCs (solid line, black triangles). ISR at sequential timepoints $(0,30$, $60 \mathrm{~min}$ ) as a function of glucose (e) or GLP-1 (f) demonstrates the dose response in ISR for both glucose and GLP-1. Ex-9 decreased ISR. $* * p<0.01$ and $* * * p<0.001$ for $\mathrm{PBH}$ participants during Ex-9 vs placebo infusion

$(p<0.05)$. Both early $\left(\mathrm{AUC}_{\mathrm{insulin}(0,60)}\right)$ and late $\left(\mathrm{AUC}_{\text {insulin }(60,180)}\right)$ insulin responses decreased by $46 \%$ with Ex-9 antagonism, bringing late-phase insulin markedly below that of NSCs (Table 2, Fig. 1b). IGI and disposition index also decreased significantly with Ex-9 infusion. Fasting ISR did not differ on placebo vs Ex-9 infusion days, but peak ISR was $71 \%$ lower with Ex-9 infusion $(p<0.001)$. Overall incremental ISR was also 59\% lower with Ex-9 infusion when adjusted for incremental unit of plasma glucose $\left(\mathrm{ISR}_{2 \mathrm{~h}} / \mathrm{G}_{2 \mathrm{~h}}\right.$ ) (Table 2, Fig. 1d). A non-linear dose dependency of ISR on glucose and GLP-1 was demonstrated with potentiation of ISR at the highest GLP-1 concentrations (Fig. 1e, f). Insulin clearance was unaffected by Ex-9 infusion (Table 2).

Incretin hormones and glucagon Ex-9 infusion did not alter GLP-1 concentrations but attenuated the relative incretin response, as measured by insulin:GLP-1 ratio, by approximately $50 \%$. Fasting and peak GIP and glucagon levels were unchanged by Ex-9 infusion, although overall glucagon $\left(\mathrm{AUC}_{\mathrm{GIP}[0,180]}\right)$ was reduced by $15 \%(p<0.01)$, while early glucagon $\left(\mathrm{AUC}_{\text {glucagon[0,60] }}\right)$ was increased by $14 \%$ during GLP-1r blockade $(p<0.05)$ (Table 3, Fig. 2a-c).

\section{Symptomatic response to $75 \mathrm{~g}$ OGTT during placebo vs Ex-9 infusion}

As shown in Table 4, Ex-9 decreased total symptom scores dramatically $(p<0.001)$. When evaluated separately during the glucose rise and fall periods, only the fall period exhibited significant score reduction. This was true for both autonomic and neuroglycopenic symptoms, which decreased by six- and tenfold $(p<0.001)$, respectively. The Ex-9 infusion was well tolerated and no adverse reactions associated with infusion of Ex-9 were reported.

\section{Pharmacokinetics of Ex-9}

Ex-9 infused at $500 \mathrm{pmol} \mathrm{kg}^{-1} \mathrm{~min}^{-1}$ over $210 \mathrm{~min}$ after a primed intravenous bolus of $7500 \mathrm{pmol} / \mathrm{kg}$ resulted in average

Table 3 Hormone responses to OGTT during i.v. placebo vs Ex-9 in PBH participants

\begin{tabular}{|c|c|c|c|}
\hline \multirow[t]{2}{*}{ Variable } & \multicolumn{2}{|l|}{$\mathrm{PBH}(n=8)$} & \multirow[b]{2}{*}{$p$ value } \\
\hline & Placebo & Ex-9 & \\
\hline \multicolumn{4}{|l|}{ GLP-1 (pmol/1) } \\
\hline Fasting & $9 \pm 0$ & $9 \pm 1$ & 0.718 \\
\hline Peak & $86 \pm 6$ & $82 \pm 14$ & 0.857 \\
\hline Time to peak (min) & $43 \pm 6$ & $39 \pm 6$ & 0.604 \\
\hline \multicolumn{4}{|l|}{$\mathrm{AUC}(\mathrm{pmol} / \mathrm{l} \mathrm{min})$} \\
\hline 0-60 min & $3270 \pm 214$ & $3268 \pm 607$ & 0.998 \\
\hline $60-180 \mathrm{~min}$ & $3136 \pm 266$ & $3621 \pm 416$ & 0.429 \\
\hline $0-180 \mathrm{~min}$ & $6406 \pm 423$ & $6889 \pm 941$ & 0.700 \\
\hline \multicolumn{4}{|l|}{ GIP (pmol/1) } \\
\hline Fasting & $14 \pm 1$ & $14 \pm 2$ & 0.824 \\
\hline Peak & $93 \pm 13$ & $87 \pm 12$ & 0.015 \\
\hline Time to peak (min) & $43 \pm 6$ & $34 \pm 4$ & 0.363 \\
\hline \multicolumn{4}{|l|}{ AUC (pmol/1 min) } \\
\hline $0-60 \mathrm{~min}$ & $3831 \pm 496$ & $3268 \pm 607$ & 0.127 \\
\hline $60-180 \mathrm{~min}$ & $3561 \pm 204$ & $3165 \pm 397$ & 0.314 \\
\hline $0-180 \mathrm{~min}$ & $7554 \pm 618$ & $6420 \pm 941$ & 0.003 \\
\hline \multicolumn{4}{|l|}{ Glucagon (ng/l) } \\
\hline Fasting & $41 \pm 3$ & $42 \pm 3$ & 0.567 \\
\hline Peak & $83 \pm 7$ & $92 \pm 6$ & 0.079 \\
\hline Time to peak (min) & $60 \pm 13$ & $45 \pm 11$ & 0.470 \\
\hline \multicolumn{4}{|l|}{ AUC (ng/l min) } \\
\hline $0-60 \mathrm{~min}$ & $3797 \pm 306$ & $4431 \pm 285$ & 0.033 \\
\hline $60-180 \mathrm{~min}$ & $8000 \pm 912$ & $8018 \pm 696$ & 0.981 \\
\hline $0-180 \mathrm{~min}$ & $11,584 \pm 1253$ & $12,019 \pm 942$ & 0.191 \\
\hline
\end{tabular}

Data are presented as mean \pm SEM

$p$ values obtained by paired two-tailed Student's $t$ test 
Fig. 2 GLP-1 (a), GIP (b) and glucagon (c) responses to an OGTT in PBH participants during placebo (solid line, black circle) vs Ex-9 (dashed line, white circle) infusion. ${ }^{* *} p<0.01$ for $\mathrm{PBH}$ participants during Ex-9 vs placebo infusion

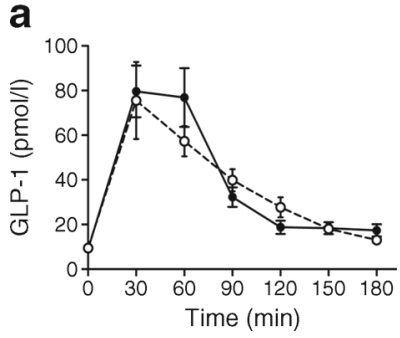

b

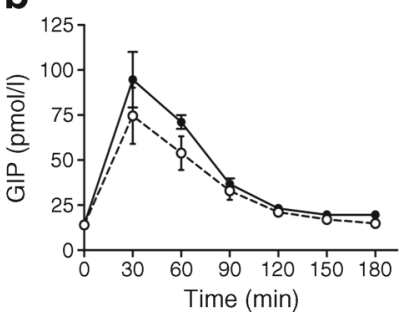

C

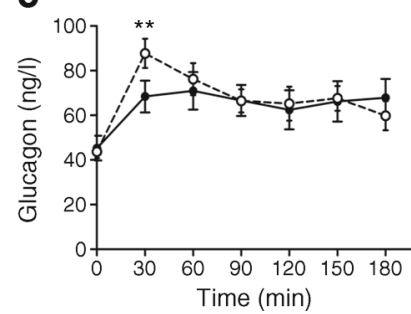

plasma concentrations of $472 \pm 17.5 \mathrm{nmol} / \mathrm{l}$, with an AUC of $47,701 \mathrm{nmol} / 1$ (ESM Fig. 1). At this infusion rate, plasma Ex-9 concentrations were approximately 4500 -fold greater than peak and AUC GLP-1 concentrations.

\section{Discussion}

This study was performed to investigate whether GLP-1 is the primary mediator of symptomatic PBH, and whether GLP-1r blockade can effectively reverse postprandial hypoglycaemia

Table 4 Symptomatic response to OGTT during i.v. infusion of placebo vs Ex-9 in PBH participants

\begin{tabular}{|c|c|c|c|c|c|}
\hline & \multicolumn{4}{|c|}{$\mathrm{PBH}(n=8)$} & \multirow[b]{2}{*}{$p$ value } \\
\hline & \multicolumn{2}{|l|}{ Placebo } & \multicolumn{2}{|c|}{ Ex-9 } & \\
\hline \multicolumn{6}{|l|}{ All symptoms } \\
\hline All timepoints & 26.5 & $(9.5)$ & 4.5 & $(7.1)$ & $<0.001$ \\
\hline Glucose rise & 8.1 & $(8.3)$ & 3.1 & $(5.0)$ & 0.191 \\
\hline Glucose fall & 24.0 & $(9.9)$ & 3.9 & $(7.2)$ & $<0.001$ \\
\hline \multicolumn{6}{|l|}{ Autonomic symptoms } \\
\hline All timepoints & 5.5 & $(3.2)$ & 0.9 & $(1.1)$ & 0.002 \\
\hline Glucose rise & 1.0 & $(1.8)$ & 0.6 & $(0.8)$ & 0.566 \\
\hline Glucose fall & 5.5 & $(3.2)$ & 1.0 & $(1.1)$ & 0.002 \\
\hline \multicolumn{6}{|c|}{ Neuroglycopenic symptoms } \\
\hline All timepoints & 18.0 & $(6.8)$ & 1.8 & $(4.6)$ & $<0.001$ \\
\hline Glucose rise & 5.1 & $(7.1)$ & 1.1 & $(3.0)$ & 0.193 \\
\hline Glucose fall & 16.4 & $(6.3)$ & 1.8 & $(4.6)$ & $<0.001$ \\
\hline \multicolumn{6}{|l|}{ Symptoms of malaise } \\
\hline All timepoints & 2.9 & $(2.9)$ & 1.9 & $(2.4)$ & 0.462 \\
\hline Glucose rise & 2.3 & (1.9) & 1.4 & (1.8) & 0.404 \\
\hline Glucose fall & 2.1 & (3.3) & 1.1 & $(2.2)$ & 0.487 \\
\hline
\end{tabular}

Data are presented as mean (SD)

Definitions: autonomic symptoms include sweating, shaking, hunger, palpitations and tremor; neuroglycopenic symptoms include blurred vision, confusion, drowsiness, odd behaviour, speech difficulty, incoordination, dizziness and inability to concentrate; symptoms of malaise include nausea and headache. Glucose rise period comprises timepoints from $\mathrm{T}=0 \mathrm{~min}$ to peak glucose; glucose fall period comprises timepoints from peak glucose to nadir glucose

$p$ values obtained by paired two-tailed Student's $t$ test and associated autonomic and neuroglycopenic symptoms. Continuous intravenous infusion of $500 \mathrm{pmol} \mathrm{kg}^{-1} \mathrm{~min}^{-1}$ of the GLP-1r antagonist Ex-9 during an OGTT achieved 100\% reversal of hypoglycaemia and a marked resolution in symptoms of hypoglycaemia in eight patients with $\mathrm{PBH}$. The plasma glucose nadir, $\leq 2.78 \mathrm{mmol} / 1$ during placebo infusion, increased by $70 \%$ during Ex-9 infusion, matching that of NSCs. Ex-9 decreased peak to nadir glucose, rate of glucose decline and $\mathrm{AUC}_{\text {glucose }(60-180)}$. Contrary to the hypothesis that hyperinsulinaemia may be driven by early and extreme elevations in plasma glucose, Ex-9 ameliorated hyperinsulinaemia despite earlier and equally high peak plasma glucose concentrations. Peak insulin responses, which during placebo infusion were $56 \%$ greater than that of NSC, were normalised during Ex-9 infusion relative to NSC. This was confirmed by various measures of beta cell function, which also decreased with GLP-1r antagonism, whereas fasting insulin and insulin clearance were unaltered.

We also sought to evaluate changes in concentrations of GIP and glucagon during GLP-1r blockade. In our eight PBH participants, GIP levels were reduced by $15 \%$ $(p<0.01)$ during Ex-9 infusion, which could have contributed to improved glucose homeostasis via further reduction of the incretin effect. Indeed, Ex-9 was shown in one study involving cultured human embryonic kidney (HEK)-293 cells to block activity both at the GLP-1r and, to a lesser degree, the GIP receptor [44]. Ex-9 may also exert indirect influences on the alpha cell: glucagon secretion is suppressed in the presence of GLP-1, thus GLP-1r blockade may disinhibit GLP-1mediated suppression of glucagon in patients with $\mathrm{PBH}$. Glucagon levels were mildly increased in the early postprandial period $(p<0.05)$; however, this did not appear to have a meaningful effect on glucose concentrations, which were not significantly elevated during the early phase relative to placebo. In addition, glucagon levels were not elevated in the late postprandial period and thus were unlikely to have prevented hypoglycaemia.

To what extent dysfunctional postprandial increases in insulin concentrations are driven by increased potentiation of incretins in the face of extraphysiological concentrations of GLP-1 warrants discussion. It is well known that the insulinotropic effects of GLP-1 are glucose and dose dependent at physiological levels of GLP-1 [45], and GLP-1-based 
therapies are known to demonstrate glucose sensitivity in such a dose-dependent manner. An effect of GLP-1 on the 'potentiation factor,' which expresses the relative potentiation of the secretory response to glucose, has been described [33], supporting the hypothesis that extraphysiological levels of GLP-1r activation may augment the secretory response to glucose. It is possible that while the insulinotropic effect of GLP-1 depends on glucose, GLP-1 action may not be restricted solely to acting on the GLP-1r. In this case, blocking the GLP-1r would not mitigate the full effect. Our data, however, reveal a non-linear dose dependency of ISR on glucose and on GLP-1 at the highest GLP-1 concentrations, which was not demonstrated during GLP-1r blockade, suggesting that the bulk of insulin secretion was mediated through GLP-1r signalling. Thus, in our opinion, excessive GLP-1 levels do not contribute in any substantial way to insulin secretion via pathways that do not involve GLP-1r signalling.

The role of GLP-1 in mediating glycaemic reductions after bariatric surgery has been a point of controversy: one study involving individuals with remission of diabetes after RYGB and healthy NSCs found that GLP-1 played a limited role, with both cohorts achieving a relatively small increase in AUC glucose during Ex-9 vs $\mathrm{NaCl}$ infusion [46], whereas one study by Salehi et al, involving symptomatic PBH patients vs asymptomatic post-RYGB controls, demonstrated that GLP-1r blockade raised the glucose nadir by $67 \%$ in symptomatic participants vs $14 \%$ in asymptomatic controls [32]. In the present study, we found a $70 \%$ increase in glucose nadir, supporting Salehi's findings, suggesting that at least in the PBH population, GLP-1 plays a major role. Salehi also reported an increase in $\mathrm{AUC}_{(0-180)}$ glucose of $200 \%$, whereas we found an increase of $21 \%$ for the same 180 min intervalresults that support but do not agree with the extent of glycaemic increase reported. Of note, during the hypoglycaemic phase (60-180 min), the AUC increased by $136 \%$. The $0-180$ min disparity may be explained by differences in: (1) the infusion rate of Ex-9 between studies (750 vs $500 \mathrm{pmol} \mathrm{kg}^{-1} \mathrm{~min}^{-1}$ ); (2) glycaemic stopping parameters (we intervened at a plasma glucose of $2.78 \mathrm{mmol} / \mathrm{l}$ ); or (3) how AUC was calculated. Furthermore, the Salehi investigation used mixed-meal tolerance tests, while the current investigation used OGTTs.

The limitations of the current study warrant consideration. The use of OGTT to study islet hormone responses to nutrient ingestion has been criticised on the basis of poor specificity. In one study, a glucose nadir of $<2.78 \mathrm{mmol} / \mathrm{l}$ following OGTT occurred in at least $10 \%$ of the normal population [47]. Also of mention is the relatively small PBH cohort in the current investigation. Nevertheless, by design this study was sufficiently powered to achieve statistical significance relating to the primary and secondary outcomes of interest, and betweensubject variability was minimised with the use of a crossover design. One limitation is that while NSCs were included in the current analysis, permitting us to demonstrate normalisation of glucose and insulin variables with GLP-1r blockade, comparison with asymptomatic surgical participants was not included. This comparison, however, has been used previously in studies $[29,32]$ that reported increased incretin and insulin responses in symptomatic vs asymptomatic post-RYGB patients.

A strength of the current study is the use of a severitygraded symptom questionnaire to assess for the presence, severity and temporal occurrence of hypoglycaemic symptoms with and without GLP-1r blockade. As more cases of this new disease have come to light, so has the recognition that symptoms of hypoglycaemia both define this disorder and are clinically meaningful. Prior studies have indicated that the presence of neuroglycopenic symptoms correlates most strongly with extent of biochemical hypoglycaemia [48]. Our results showed that in concert with normalising insulin and glucose concentrations, Ex-9 globally reduced the presence and severity of all hypoglycaemic symptoms and almost completely prevented the occurrence of neuroglycopenic symptoms which, to our knowledge, we are the first to report. This reversal has major implications for health, safety and quality of life. This study is also the first to reveal a non-linear doseresponse relationship between ISR and both glucose and GLP-1 at higher concentrations in PBH participants, which may explain the severity of hypoglycaemia in affected patients as a function of increased GLP-1 secretion, and its reversal with GLP-1r blockade.

In summary, our findings support a critical role for GLP-1 in mediating symptomatic $\mathrm{PBH}$ and provide evidence for the effective use of pharmacological GLP-1r blockade. In the current investigation, intravenous infusion of Ex-9 normalised insulin secretion, thereby preventing hypoglycaemia and substantially reducing the autonomic and neuroglycopenic symptoms that characterise this disorder. For this generally young patient population with frequent neuroglycopenic outcomes, the long-term toll on health and quality of life is potentially immense. In the absence of current pharmacological approaches, and given the demonstrated efficacy of GLP-1r blockade to remedy PBH and neuroglycopenic symptoms, competitive antagonism at the GLP-1r merits consideration for development as an effective therapeutic strategy.

Acknowledgements We thank the nursing and laboratory staff from the Clinical and Translational Research Unit, Stanford University School of Medicine, and L. Albæk from the Panum Institute, University of Copenhagen, Denmark, for their skilled technical expertise. We are ever grateful to our research study participants.

Data availability The published data generated during and/or analysed during the current study are available from the corresponding author on reasonable request. 
Funding The authors gratefully acknowledge research support from the National Institutes of Health, including KL2 TR 001083, UL1 TR 001085 and L30 TR001569-01 (to CMC); and from the Translational Research and Applied Medicine (TRAM) Program of Stanford University School of Medicine (to CMC).

Duality of interest The authors declare that there is no duality of interest associated with this manuscript.

Contribution statement All authors contributed substantially to this work. CMC and TLM designed the study, acquired the data, performed the statistical analyses, interpreted the data, drafted the manuscript and approved the final content. L-FL and CFD performed laboratory assays and analyses. L-FL, CFD and JJH assisted in interpretation of data and provided critical review of the article. All authors approved the final version to be published. TLM is responsible for the integrity of the work as a whole.

\section{References}

1. Ogden C, Carroll M, Kit B, Flegal K (2014) Prevalence of childhood and adult obesity in the United States, 2011-2012. JAMA 311: 806-814

2. Finkelstein EA, Trogdon JG, Cohen JW, Dietz W (2009) Annual medical spending attributable to obesity: payer- and servicespecific estimates. Health Aff 28:822-831

3. Sjostrom L, Lindroos AK, Peltonen M et al (2004) Lifestyle, diabetes, and cardiovascular risk factors 10 years after bariatric surgery. N Engl J Med 351:2683-2693

4. Adams TD, Gress RE, Smith SC et al (2007) Long-term mortality after gastric bypass surgery. N Engl J Med 357:753-761

5. Pories WJ, Swanson MS, MacDonald KG et al (1995) Who would have thought it? An operation proves to be the most effective therapy for adult-onset diabetes mellitus. Ann Surg 222:339-350

6. Schauer PR, Burguera B, Ikramuddin S et al (2003) Effect of laparoscopic Roux-en Y gastric bypass on type 2 diabetes mellitus. Ann Surg 238:467-484

7. Rubino F, Nathan D, Eckel R et al (2016) Metabolic surgery in the treatment algorithm for type 2 diabetes: a joint statement by international diabetes organizations. Diabetes Care 39:861-877

8. Kashyap SR, Bhatt DL, Wolski K et al (2013) Metabolic effects of bariatric surgery in patients with moderate obesity and type 2 diabetes: analysis of a randomized control trial comparing surgery with intensive medical treatment. Diabetes Care 36:2175-2182

9. Korner J, Bessler M, Inabnet W, Taveras C, Holst JJ (2007) Exaggerated glucagon-like peptide- 1 and blunted glucosedependent insulinotropic peptide secretion are associated with Roux-en-Y gastric bypass but not adjustable gastric banding. Surg Obes Relat Dis 3:597-601

10. Holst J, Madsbad S (2016) Mechanisms of surgical control of type 2 diabetes: GLP-1 is key factor. Surg Obes Relat Dis 12:1236-1242

11. Vidal J, De Hollanda A, Jiménez A (2016) GLP-1 is not the key mediator of the health benefits of metabolic surgery. Surg Obes Relat Dis 12:1225-1229

12. Marsk R, Jonas E, Rasmussen F, Naslund E (2010) Nationwide cohort study of post-gastric bypass hypoglycaemia including 5,040 patients undergoing surgery for obesity in 1986-2006 in Sweden. Diabetologia 53:2307-2311

13. Kellog TA, Bantle JP, Leslie DB et al (2008) Postgastric bypass hyperinsulinemic hypoglycaemia syndrome: characterization and response to a modified diet. Surg Obes Relat Dis 4:492-499
14. Gribsholt SB, Pedersen AM, Svensson E, Thomsen RW, Richelsen B (2016) Prevalence of self-reported symptoms after gastric bypass surgery for obesity. JAMA Surg 151:504-511

15. Kefurt R, Langer FB, Schindler K et al (2015) Hypoglycemia after Roux-En-Y gastric bypass: detection rates of continuous glucose monitoring (CGM) versus mixed meal test. Surg Obes Relat Dis 11: 564-569

16. Valderas J, Ahuad J, Rubio L, Escalona M, Pollak F, Maiz A (2012) Acarbose improves hypoglycaemia following gastric bypass surgery without increasing glucagon-like peptide 1 levels. Obes Surg 22:582-586

17. Spanakis E, Gragnoli C (2009) Successful medical management of status post-Roux-en-Y-gastric-bypass hyperinsulinemic hypoglycaemia. Obes Surg 19:1333-1334

18. Moreira R, Moreira R, Machado N, Gonçalves T, Coutinho W (2008) Post-prandial hypoglycaemia after bariatric surgery: pharmacological treatment with verapamil and acarbose. Obes Surg 18: 1618-1621

19. McLaughlin TL, Peck M, Holst JJ, Deacon C (2010) Reversible hyperinsulinemic hypoglycaemia after gastric bypass: a consequence of altered nutrient delivery. J Clin Endocrinol Metab 95: $1851-1855$

20. Craig C, Lamendola C, Deacon C, Holst J, McLaughlin T (2015) The use of gastrostomy tube for the long-term remission of hyperinsulinemic hypoglycaemia after Roux-en-Y gastric bypass - a case report. AACE Clin Case Rep 1:e84-e87

21. Fernández-Esparrach G, Lautz D, Thompson C (2016) Peroral endoscopic anastomotic reduction improves intractable dumping syndrome in Roux-en-Y gastric bypass patients. Surg Obes Relat Dis 6: 36-40

22. Lee CJ, Brown T, Magnuson TH et al (2013) Hormonal response to a mixed-meal challenge after reversal of gastric bypass for hypoglycaemia. J Clin Endocrinol Metab 98:1208-1212

23. Finks JF, Osborne NH, Birkmeyer JD (2011) Trends in hospital volume and operative mortality for high-risk surgery. N Engl J Med 364:2128-2137

24. Service GJ, Thompson GB, Service FJ, Andrews JC, CollazoClavell ML, Lloyd RV (2005) Hyperinsulinemic hypoglycaemia with nesidioblastosis after gastric-bypass surgery. N Engl J Med 353:249-254

25. Kim SH, Abbasi F, Lamendola C, Reaven GM, McLaughlin T (2010) Glucose-stimulated insulin secretion in gastric bypass patients with hypoglycaemic syndrome: no evidence for inappropriate pancreatic beta cell function. Obes Surg 20:1110-1116

26. Patti ME, McMahon G, Mun EC et al (2005) Severe hypoglycaemia post-gastric bypass requiring partial pancreatectomy: evidence for inappropriate insulin secretion and pancreatic islet hyperplasia. Diabetologia 48:2236-2240

27. Falken Y, Hellstrom PM, Holst JJ, Nasund E (2011) Changes in glucose homeostasis after Roux-en-Y gastric bypass surgery for obesity at day three, two months, and one year after surgery: role of gut peptides. J Clin Endocrinol Metab 96:2227-2235

28. Marathe CS, Rayner CK, Jones KL, Horowitz M (2013) Relationships between gastric emptying, postprandial glycemia, and incretin hormones. Diabetes Care 36:1396-1405

29. Goldfine A, Mun E, Devine E et al (2007) Patients with neuroglycopenia after gastric bypass surgery have exaggerated incretin and insulin secretory responses to a mixed meal. J Clin Endocrinol Metab 92:4678-4685

30. Malin SK, Kashyap SR (2015) Differences in weight loss and gut hormones: Roux-en-Y gastric bypass and sleeve gastrectomy surgery. Curr Obes Rep 4:279-286

31. Salehi M, Prigeon RL, D'Alessio DA (2011) Gastric bypass surgery enhances glucagon-like peptide-1 stimulated postprandial insulin secretion in humans. Diabetes 60:2308-2314 
32. Salehi M, Gastaldelli A, D'Alessio D (2014) Blockade of glucagonlike peptide 1 receptor corrects postprandial hypoglycaemia after gastric bypass. Gastroenterology 146:669-680

33. Ahren B, Holst JJ, Mari A (2003) Characterization of GLP-1 effects on beta-cell function after meal ingestion in humans. Diabetes Care 26:2860-2864

34. Deary IJ, Hepburn DA, MacLeod KM, Frier BM (1993) Partitioning the symptoms of hypoglycaemia using multi-sample confirmatory factor analysis. Diabetologia 36:771-777

35. Hepburn DA, Deary IJ, Frier BM, Patrick AW, Quinn JD, Fisher BM (1991) Symptoms of acute insulin-induced hypoglycaemia in humans with and without IDDM. Diabetes Care 14:949-957

36. Orskov C, Rabenhøj L, Wettergren A, Kofod H, Holst JJ (1994) Tissue and plasma concentrations of amidated and glycineextended glucagon-like peptide-1 in humans. Diabetes 43:535-539

37. Lindgren O, Carr RD, Deacon CF et al (2011) Incretin hormone and insulin responses to oral vs intravenous lipid administration in humans. J Clin Endocrinol Metab 96:2519-2524

38. Kielgast U, Holst JJ, Madsbad S (2011) Antidiabetic actions of endogenous and exogenous GLP-1 in type 1 diabetic patients with and without residual $\beta$-cell function. Diabetes 60:1599-1607

39. Matthews DR, Hosker JP, Rudenski AS, Naylor BA, Treacher DF, Turner RC (1985) Homeostasis model assessment: insulin resistance and beta cell function from fasting plasma glucose and insulin concentrations in man. Diabetologia 28:412-419

40. Utzschneider KM, Prigeon RL, Carr DB et al (2006) Impact of differences in fasting glucose and glucose tolerance on the hyperbolic relationship between insulin sensitivity and insulin responses. Diabetes Care 29:356-362

41. Hovorka R, Soons PA, Young MA (1996) ISEC: a program to calculate insulin secretion. Comput Methods Prog Biomed 50: 253-264

42. Van Cauter E, Mestrez F, Sturis J, Polonsky KS (1992) Estimation of insulin secretion rates from C-peptide levels. Comparison of individual and standard kinetic parameters for $\mathrm{C}$-peptide clearance. Diabetes 41:368-377

43. Mari A, Schmitz O, Gastaldelli A, Oestergaard T, Nyholm B, Ferrannini E (2002) Meal and oral glucose tests for assessment of beta-cell function: modeling analysis in normal subjects. Am J Physiol Endocrinol Metab 283:E1159-E1166

44. Al-Sabah S, Al-Fulaij M, Ahmed HA (2014) Selectivity of peptide ligands for the human incretin receptors expressed in HEK-293 cells. Eur J Pharmacol 741:311-315

45. Kjems LL, Holst JJ, Volund A, Madsbad S (2003) The influence of GLP-1 on glucose-stimulated insulin secretion: effects on beta-cell sensitivity in type 2 and nondiabetic subjects. Diabetes 52:380-386

46. Jimenez A, Casamitjana R, Viaplana-Masclans J, Lacy A, Vidal J (2013) GLP-1 action and glucose tolerance in participants with remission of type 2 diabetes after gastric bypass surgery. Diabetes Care 36:2062-2069

47. Lev-Ran A, Anderson RW (1981) The diagnosis of postprandial hypoglycaemia. Diabetes 30:996-999

48. Mitrakou A, Ryan C, Veneman T et al (1991) Hierarchy of glycaemic thresholds for counterregulatory hormone secretion, symptoms, and cerebral dysfunction. Am J Physiol 260:E67-E74 